\title{
ANALYZING THE GROUP SPARSITY BASED ON THE RANK MINIMIZATION METHODS
}

\author{
Zhiyuan Zha ${ }^{\dagger}$, Xin Liu ${ }^{\ddagger}$, Xiaohua Huang ${ }^{\ddagger}$, Henglin Shi ${ }^{\ddagger}$, Yingyue X $u^{\ddagger}$, Qiong Wang ${ }^{\dagger}, *$, Lan Tang ${ }^{\dagger}$, Xinggan Zhang ${ }^{\dagger}$ \\ $\dagger^{\dagger}$ School of Electronic Science and Engineering, Nanjing University, Nanjing 210023, China. \\ $\ddagger$ The Center for Machine Vision and Signal Analysis, University of Oulu, 90014, Finland.
}

\begin{abstract}
Sparse coding has achieved a great success in various image processing studies. However, there is not any benchmark to measure the sparsity of image patch/group because sparse discriminant conditions cannot keep unchanged. This paper analyzes the sparsity of group based on the strategy of the rank minimization. Firstly, an adaptive dictionary for each group is designed. Then, we prove that group-based sparse coding is equivalent to the rank minimization problem, and thus the sparse coefficients of each group are measured by estimating the singular values of each group. Based on that measurement, the weighted Schatten $p$-norm minimization (WSNM) has been found to be the closest solution to the real singular values of each group. Thus, WSNM can be equivalently transformed into a non-convex $\ell_{p}$-norm minimization problem in group-based sparse coding. Experimental results on two applications: image inpainting and image compressive sensing (CS) recovery show that the proposed scheme outperforms many state-of-the-art methods.
\end{abstract}

Index Terms - group sparsity, rank minimization, the weighted schatten $p$-norm, $\ell_{p}$-norm, adaptive dictionary.

\section{INTRODUCTION}

Traditional patch-based sparse coding assumes that each patch of an image can be precisely modeled as a sparse linear combination of basic elements. It has been successfully used in various image processing applications $[1,2,3]$. However, patch-based sparse coding model of natural images usually suffers from some limits, such as dictionary learning with great computational complexity, neglecting the relationships among similar patches.

Instead of using patch as the basic unit of sparse coding, group-based sparse coding can offer a powerful mechanism of combining local sparsity and nonlocal self-similarity of images simultaneously in a unified framework $[4,5]$. To be concrete, an image $\boldsymbol{X}$ with size $N$ is divided into $n$ overlapped patches of size $\sqrt{d} \times \sqrt{d}$, and each patch is denoted by the vector $\boldsymbol{x}_{i} \in \Re^{d}, i=1,2, \ldots, n$. Then for each patch $\boldsymbol{x}_{i}$, its $k$ similar patches are selected from a $I \times I$ sized search window to form a set $\boldsymbol{S}_{i}$. After this, all the patches in $\boldsymbol{S}_{i}$ are stacked

\footnotetext{
* Corresponding Author.
}

into a matrix $\boldsymbol{X}_{i} \in \Re^{d \times k}$, i.e., $\boldsymbol{X}_{i}=\left\{\boldsymbol{x}_{i, 1}, \boldsymbol{x}_{i, 2}, \ldots, \boldsymbol{x}_{i, k}\right\}$. The matrix $\boldsymbol{X}_{i}$ consisting of all the patches with similar structures is called a group, where $\boldsymbol{x}_{i, k}$ denotes the $k$-th similar patch of the $i$-th group. Similar to patch-based sparse coding [1], given a dictionary $\boldsymbol{D}_{i}$, each group $\boldsymbol{X}_{i}$ can be sparsely represented as $\boldsymbol{\alpha}_{i}=\boldsymbol{D}_{i}^{-1} \boldsymbol{X}_{i}$ and solved by the following $\ell_{0}$-norm minimization problem,

$$
\boldsymbol{\alpha}_{i}=\arg \min _{\boldsymbol{\alpha}_{i}}\left\{\frac{1}{2}\left\|\boldsymbol{X}_{i}-\boldsymbol{D}_{i} \boldsymbol{\alpha}_{i}\right\|_{F}^{2}+\lambda\left\|\boldsymbol{\alpha}_{i}\right\|_{0}\right\}
$$

where $\lambda$ is the regularization parameter, $\|*\|_{F}^{2}$ denotes the Frobenius norm, and $\|*\|_{0}$ is $\ell_{0}$-norm, counting the non-zero entries of $\boldsymbol{\alpha}_{i}$.

However, since $\|*\|_{0}$ norm minimization is a difficult combinatorial optimization problem, solving Eq. (1) is NPhard. For this reason, it is often replaced by the $\ell_{1}$-norm or the reweighted $\ell_{1}$-norm to make the optimization problem easy [6]. Nonetheless, the solution of these norm minimizations is only the estimation of the real sparsity solution under certain conditions. For instance, Candès et al. [7] proposed that solving $\ell_{1}$-norm optimization problem can recover a $K$ sparse signal $\boldsymbol{x} \in \Re^{N}$ from $M=O(K \log (N / K))$ random measurements in compressive sensing (CS). However, the $\ell_{1}$ norm minimization cannot still obtain the real sparsity solution, one important reason is nobody can guarantee the invariance of sparse discriminant conditions. In other words, there is not any benchmark to measure the sparsity of a signal.

With the above consideration, we analyze the group sparsity from the point of the rank minimization. To the best of our knowledge, few works have exploited the rank minimization methods to analyze the sparsity of image groups. The contribution of this paper is as follows. First, an adaptive dictionary for each group is designed with a low computational complexity, rather than dictionary learning from natural images. Second, based on this dictionary learning scheme, we prove the equivalence of group-based sparse coding and the rank minimization problem, and thus the sparse coefficients of each group are measured by calculating the singular values of each group. Thus, we have a benchmark to measure the sparsity of each group because the singular values of the original image group can be easily computed by SVD operator. Third, we exploit four nuclear norms (i.e., standard nuclear norm [8], the weighted nuclear norm [9], Schatten $p$-norm 
[10] and the weighted Schatten $p$-norm [11]) to analyze the sparsity of each group and the solution of the weighted Schatten $p$-norm minimization (WSNM) is the nearest to real singular values of each group. Therefore, WSNM is equivalently turned into a non-convex $\ell_{p}$-norm minimization problem in group-based sparse coding. Experimental results on two lowlevel vision tasks, i.e., image inpainting and image compressive sensing (CS) recovery have demonstrated that the proposed scheme outperforms many state-of-the-art schemes.

\section{BACKGROUND}

\subsection{Rank minimization method}

The main goal of low rank matrix approximation (LRMA) is to recover the underlying low rank structure from its degraded/corrupted observed version. In general, methods of LRMA can be classified into two categories: the low rank matrix factorization (LRMF) methods $[12,13]$ and the rank minimization methods $[8,9,10,11]$. In this work we focus on the latter category. More specifically, given an input matrix $\boldsymbol{Y}$, the rank minimization methods aim to find a low rank matrix $\boldsymbol{X}$, which is as close to $\boldsymbol{Y}$ as possible under $F$-norm data fidelity and one nuclear norm,

$$
\hat{\boldsymbol{X}}=\arg \min _{\boldsymbol{X}}\|\boldsymbol{Y}-\boldsymbol{X}\|_{F}^{2}+\lambda \boldsymbol{R}(\boldsymbol{X})
$$

where $\lambda$ is a trade-off parameter between the loss function and the low rank regularization induced by one nuclear norm $\boldsymbol{R}(\boldsymbol{X})$. We will briefly introduce several nuclear norms including standard nuclear norm [8], the weighted nuclear norm [9], Schatten $p$-norm [10] and the weighted Schatten $p$-norm [11] in the next subsection.

\subsection{Nuclear norms}

In this subsection, we first introduce the weighted Schatten $p$-norm [11] of a matrix $\boldsymbol{X} \in \Re^{m \times n}$, which is defined as

$$
\|\boldsymbol{X}\|_{\boldsymbol{w}, S_{p}}=\left(\sum_{i=1}^{\min \{m, n\}} w_{i} \sigma_{i}^{p}\right)^{\frac{1}{p}}
$$

where $0<p \leq 1$, and $\sigma_{i}$ is the $i$-th singular value of $\boldsymbol{X}$. $\boldsymbol{w}=\left[w_{1}, \ldots, w_{\min \{m, n\}}\right]$ and $w_{i} \geq 0$ is a non-negative weight assigned to $\sigma_{i}$. Then the weighted Schatten $p$-norm of $\boldsymbol{X}$ with power $p$ is

$$
\|\boldsymbol{X}\|_{\boldsymbol{w}, S_{p}}^{p}=\sum_{i=1}^{\min \{m, n\}} w_{i} \sigma_{i}^{p}=\operatorname{Tr}\left(\boldsymbol{W} \boldsymbol{\Delta}^{p}\right)
$$

where $\boldsymbol{W}$ and $\boldsymbol{\Delta}$ are diagonal matrices whose diagonal entries are composed of $w_{i}$ and $\sigma_{i}$, respectively.

The Schatten $p$-norm [10] of a matrix $\boldsymbol{X}$ can be represented by setting $\boldsymbol{w}=[1,1, \ldots, 1]$ in Eq. (3),

$$
\|\boldsymbol{X}\|_{S_{p}}=\left(\sum_{i=1}^{\min \{m, n\}} \sigma_{i}^{p}\right)^{\frac{1}{p}}=\left(\operatorname{Tr}\left(\left(\boldsymbol{X}^{T} \boldsymbol{X}\right)^{\frac{p}{2}}\right)\right)^{\frac{1}{p}}
$$

The weighted nuclear norm [9] of a matrix $\boldsymbol{X}$ can be represented by setting $p=1$ in Eq. (3),

$$
\|\boldsymbol{X}\|_{\boldsymbol{w}, *}=\left(\sum_{i=1}^{\min \{m, n\}} w_{i} \sigma_{i}\right)=\operatorname{Tr}(\boldsymbol{W} \boldsymbol{\Delta})
$$

A widely used standard nuclear norm [8] of a matrix $\boldsymbol{X}$ can be represented by setting $p=1$ and $\boldsymbol{w}=[1,1, \ldots, 1]$ in Eq. (3),

$$
\|\boldsymbol{X}\|_{*}=\sum_{i=1}^{\min \{m, n\}} \sigma_{i}=\operatorname{Tr}\left(\left(\boldsymbol{X}^{T} \boldsymbol{X}\right)^{\frac{1}{2}}\right)
$$

\section{ANALYZING THE SPARSITY OF GROUP BASED ON THE RANK MINIMIZATION METHODS}

Since the sparse discriminant conditions cannot keep unchanged, there is not any benchmark to measure the sparsity of image group. Therefore, we analyze the group sparsity from the point of the rank minimization. To this end, an adaptive dictionary for each group is designed with a low computational complexity, rather than dictionary learning from natural images. Based on this dictionary learning scheme, we prove the equivalence of group-based sparse coding and the rank minimization problem, i.e., the sparse coefficients of each group are measured by calculating the singular values of each group. Therefore, we possess a benchmark to measure the sparsity of each group by rank minimization methods since the singular values of the original image group can be easily obtained. In this way, we can achieve a clear visual comparison effect to analyze the sparsity of each group based on the rank minimization methods (See Fig. 1).

\subsection{Adaptive dictionary learning}

In this subsection, an adaptive dictionary learning method is designed, that is, for each group $\boldsymbol{X}_{i}$, its adaptive dictionary can be learned from its observation $\boldsymbol{Y}_{i} \in \Re^{d \times k}$.

More specifically, we apply the singular value decomposition (SVD) to $\boldsymbol{Y}_{i}$,

$$
\boldsymbol{Y}_{i}=\boldsymbol{U}_{i} \boldsymbol{\Sigma}_{i} \boldsymbol{V}_{i}^{T}=\sum_{j=1}^{m} \boldsymbol{\sigma}_{i, j} \boldsymbol{u}_{i, j} \boldsymbol{v}_{i, j}^{T}
$$

where $\boldsymbol{\mu}_{i}=\left[\boldsymbol{\sigma}_{i, 1}, \boldsymbol{\sigma}_{i, 2}, \ldots, \boldsymbol{\sigma}_{i, m}\right], m=\min (d, k), \boldsymbol{\Sigma}_{i}=$ $\operatorname{diag}\left(\boldsymbol{\mu}_{i}\right)$ is a diagonal matrix whose non-zero elements are represented by $\boldsymbol{\mu}_{i}$, and $\boldsymbol{u}_{i, j}, \boldsymbol{v}_{i, j}$ are the columns of $\boldsymbol{U}_{i}$ and $\boldsymbol{V}_{i}$, respectively.

Moreover, we define each dictionary atom $\boldsymbol{d}_{i, j}$ of the adaptive dictionary $\boldsymbol{D}_{i}$ for each group $\boldsymbol{Y}_{i}$ as follows:

$$
\boldsymbol{d}_{i, j}=\boldsymbol{u}_{i, j} \boldsymbol{v}_{i, j}^{T}, \quad j=1,2, \ldots, m
$$

Finally, by learning an adaptive dictionary $\boldsymbol{D}_{i}=$ $\left[\boldsymbol{d}_{i, 1}, \boldsymbol{d}_{i, 2}, \ldots, \boldsymbol{d}_{i, m}\right]$ from each group $\boldsymbol{Y}_{i}$. The proposed dictionary learning method is efficient due to the fact that it only requires one SVD operator for each group. 


\subsection{Prove the equivalence of group-based sparse coding and the rank minimization problem}

To prove that the group-based sparse coding is equivalent to the rank minimization problem, we firstly give two following lemmas.

Lemma 1 The minimization problem

$$
\boldsymbol{x}=\arg \min _{\boldsymbol{x}} \frac{1}{2}\|\boldsymbol{x}-\boldsymbol{a}\|_{2}^{2}+\tau \cdot\|\boldsymbol{x}\|_{1}
$$

has a closed form, which can be expressed as

$$
\hat{\boldsymbol{x}}=\operatorname{soft}(\boldsymbol{a}, \tau)=\operatorname{sgn}(\boldsymbol{a}, \tau) \cdot \max (a b s(\boldsymbol{a})-\tau, 0)
$$

Proof: see [14].

Consider the SVD of a matrix $\boldsymbol{P} \in \Re^{n_{1} \times n_{2}}$ of rank $r$

$$
\boldsymbol{P}=\boldsymbol{U} \boldsymbol{\Sigma} \boldsymbol{V}^{T}, \boldsymbol{\Sigma}=\operatorname{diag}\left(\left\{\sigma_{i}\right\}_{1 \leq i \leq r}\right)
$$

where $\boldsymbol{U} \in \Re^{n_{1} \times r}$ and $\boldsymbol{V} \in \Re^{n_{2} \times r}$ are orthogonal matrices, respectively. $\sigma_{i}$ is the $i$-th singular value of $\boldsymbol{P}$. For each $\tau \geq 0$, the soft-thresholding operator $\mathcal{D}_{\tau}$ is defined as

$$
\mathcal{D}_{\tau}(\boldsymbol{P})=\boldsymbol{U D}_{\tau}(\boldsymbol{\Sigma}) \boldsymbol{V}^{T}, \quad \mathcal{D}_{\tau}(\boldsymbol{\Sigma})=\operatorname{soft}\left(\sigma_{i}, \tau\right)
$$

Lemma 2 For each $\tau \geq 0$, and $\boldsymbol{Q} \in \Re^{n_{1} \times n_{2}}$,the singular value shrinkage operator Eq. (13) satisfies

$$
\mathcal{D}_{\tau}(\boldsymbol{Q})=\arg \min _{\boldsymbol{P}}\left\{\frac{1}{2}\|\boldsymbol{P}-\boldsymbol{Q}\|_{F}^{2}+\tau\|\boldsymbol{P}\|_{*}\right\}
$$

Proof: see [8].

Now, the classical $\ell_{1}$-norm group-based sparse coding problem can be represented as

$$
\boldsymbol{\alpha}_{i}=\arg \min _{\boldsymbol{\alpha}_{i}}\left\{\frac{1}{2}\left\|\boldsymbol{Y}_{i}-\boldsymbol{D}_{i} \boldsymbol{\alpha}_{i}\right\|_{F}^{2}+\lambda\left\|\boldsymbol{\alpha}_{i}\right\|_{1}\right\}
$$

According to the above design of adaptive dictionary $\boldsymbol{D}_{i}$, we have the following conclusion.

\section{Theorem 1}

$$
\left\|\boldsymbol{Y}_{i}-\boldsymbol{X}_{i}\right\|_{F}^{2}=\left\|\boldsymbol{\mu}_{i}-\boldsymbol{\alpha}_{i}\right\|_{2}^{2}
$$

where $\boldsymbol{Y}_{i}=\boldsymbol{D}_{i} \boldsymbol{\mu}_{i}$ and $\boldsymbol{X}_{i}=\boldsymbol{D}_{i} \boldsymbol{\alpha}_{i}$.

Proof: see supplementary material.

\section{Theorem 2}

The equivalence of the group-based sparse coding and the rank minimization problem is satisfied under the adaptive dictionary $\boldsymbol{D}_{i}$.

Proof: see supplementary material.

It can be similarly proven that the reweighted $\ell_{1}$-norm and $\ell_{p}$-norm minimization are equivalent to the weighted nuclear norm minimization (WNNM) [10] and the weighted Schatten $p$-norm minimization (WSNM) [11], respectively.

Note that the main difference between sparse coding and the rank minimization problem is that sparse coding has a dictionary learning operator and the rank minimization problem does not involve.

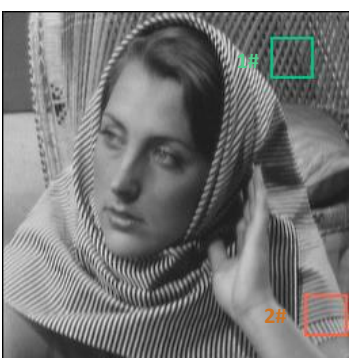

(a) Original image

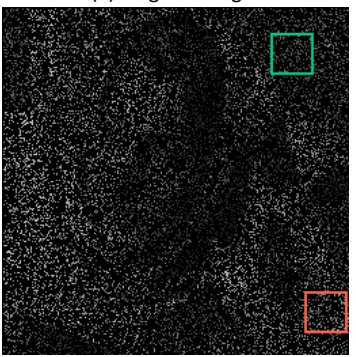

(b) $80 \%$ pixels misisng

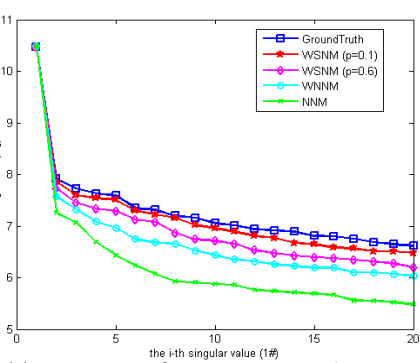

(c) comparison of NNM, WNNM and WSNM

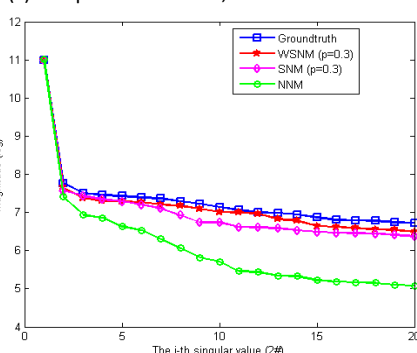

(d) comparison of NNM, SNM and WSNM
Fig. 1. Analyzing the sparsity of each group based nuclear norms minimization.

\subsection{Analyzing the sparsity of group based on the nuclear norms minimization}

Based on Theorem 2, the group-based sparse coding can be turned into the rank minimization problem. Now, four nuclear norms are used to constrain Eq. (2) to analyze the sparsity of each group, i.e., nuclear norm minimization (NNM) [8], the weighted nuclear norm minimization (WNNM) [9], Schatten $p$-norm minimization (SNM) [10] and the weighted Schatten $p$-norm minimization (WSNM) [11]. In these experiments, a gray image Barbara is used as an example in the context of image inpainting, where $80 \%$ pixels are damaged in Fig. 1(b). We generate two groups based on 1\# position and 2\# position which are shown in Fig. 1(a). As shown in Fig. 1(c) and Fig. 1(d), it can be seen that the singular values of WSNM result are the best approximation to the groundtruth in comparison with other methods. Therefore, based on Theorem 2, WSNM can be equivalently transformed into solving the non-convex $\ell_{p}$-norm minimization to measure the sparsity in group-based sparse coding.

\section{IMAGE RESTORATION USING GROUP-BASED SPARSE CODING WITH NON-CONVEX $\ell_{P}$-NORM MINIMIZATION}

In this section, we verify the proposed scheme in the application of image restoration (IR). IR aims to reconstruct a high quality image $\boldsymbol{X}$ from its degraded observation $\boldsymbol{Y}$,

$$
\boldsymbol{Y}=\boldsymbol{H} \boldsymbol{X}+\boldsymbol{N}
$$

where $\boldsymbol{H}$ is the degraded operator and $\boldsymbol{N}$ is usually assumed to be additive white Gaussian noise. In this paper, we will fo- 
cus on image inpainting and image compressive sensing (CS) recovery.

In the scenario of IR, what we observed is the degraded image $\boldsymbol{Y}$ via Eq. (17), and thus the goal is to employ the proposed scheme to recover the original image $\boldsymbol{X}$ from $\boldsymbol{Y}$ by solving the following non-convex $\ell_{p}$-norm minimization problem,

$$
\boldsymbol{\alpha}=\arg \min _{\boldsymbol{\alpha}} \frac{1}{2}\|\boldsymbol{Y}-\boldsymbol{H D} \boldsymbol{\alpha}\|_{2}^{2}+\lambda\|\boldsymbol{w} \boldsymbol{\alpha}\|_{p}
$$

\subsection{ADMM based for non-convex $\ell_{p}$-norm minimization}

Solving the objective function of Eq. (18) is very difficult, since it is a large scale non-convex optimization problem. To make the proposed scheme tractable and robust, in this paper we present the alternating direction method of multipliers (ADMM) [15] to solve Eq. (18). Specifically, we introduce an auxiliary variable $\boldsymbol{Z}$ and Eq. (18) can be rewritten as

$$
\boldsymbol{\alpha}=\arg \min _{\boldsymbol{Z}, \boldsymbol{\alpha}} \frac{1}{2}\|\boldsymbol{Y}-\boldsymbol{H Z}\|_{2}^{2}+\lambda\|\boldsymbol{w} \boldsymbol{\alpha}\|_{p}, \text { s.t. } \boldsymbol{Z}=\boldsymbol{D} \boldsymbol{\alpha}
$$

Therefore, Eq. (19) can be translated into three iterative steps:

$$
\begin{gathered}
\boldsymbol{Z}^{t+1}=\arg \min _{\boldsymbol{Z}} \frac{1}{2}\|\boldsymbol{Y}-\boldsymbol{H Z}\|_{2}^{2}+\frac{\rho}{2}\left\|\boldsymbol{Z}-\boldsymbol{D} \boldsymbol{\alpha}^{t}-\boldsymbol{C}^{t}\right\|_{2}^{2} \\
\boldsymbol{\alpha}^{t+1}=\arg \min _{\boldsymbol{\alpha}} \lambda\|\boldsymbol{w} \boldsymbol{\alpha}\|_{p}+\frac{\rho}{2}\left\|\boldsymbol{Z}^{t+1}-\boldsymbol{D} \boldsymbol{\alpha}-\boldsymbol{C}^{t}\right\|_{2}^{2}
\end{gathered}
$$

and

$$
\boldsymbol{C}^{t+1}=\boldsymbol{C}^{t}-\left(\boldsymbol{Z}^{t+1}-\boldsymbol{D} \boldsymbol{\alpha}^{t+1}\right)
$$

It can be seen that the minimization for Eq. (19) involves splitting two minimization sub-problems, i.e., $\boldsymbol{Z}$ and $\boldsymbol{\alpha}$ subproblem. Next, we will show that there is an efficient solution to each sub-problem. To avoid confusion, the subscribe $t$ may be omitted for conciseness.

\subsubsection{Z sub-problem}

Given $\boldsymbol{\alpha}$, the $\boldsymbol{Z}$ sub-problem denoted by Eq. (20) becomes

$$
\min _{\boldsymbol{Z}} \boldsymbol{L}_{1}(\boldsymbol{Z})=\min _{\boldsymbol{Z}} \frac{1}{2}\|\boldsymbol{Y}-\boldsymbol{H} \boldsymbol{Z}\|_{2}^{2}+\frac{\rho}{2}\|\boldsymbol{Z}-\boldsymbol{D} \boldsymbol{\alpha}-\boldsymbol{C}\|_{2}^{2}
$$

Clearly, Eq. (23) has a closed-form solution and its solution can be expressed as

$$
\hat{\boldsymbol{Z}}=\left(\boldsymbol{H}^{T} \boldsymbol{H}+\rho \boldsymbol{I}\right)^{-1}\left(\boldsymbol{H}^{T} \boldsymbol{Y}+\rho(\boldsymbol{D} \boldsymbol{\alpha}+\boldsymbol{C})\right)
$$

where $I$ represents the identity matrix.

\subsection{2. $\alpha$ sub-problem}

Given $\boldsymbol{Z}$, similarity, according to Eq. (21), the $\boldsymbol{\alpha}$ sub-problem can be written as

$$
\min _{\boldsymbol{\alpha}} \boldsymbol{L}_{2}(\boldsymbol{\alpha})=\min _{\boldsymbol{\alpha}} \frac{1}{2}\|\boldsymbol{D} \boldsymbol{\alpha}-\boldsymbol{R}\|_{2}^{2}+\frac{\lambda}{\rho}\|\boldsymbol{w} \boldsymbol{\alpha}\|_{p}
$$

where $\boldsymbol{R}=\boldsymbol{Z}-\boldsymbol{C}$.

However, due to the complex structure of $\|\boldsymbol{w} \boldsymbol{\alpha}\|_{p}$, it is difficult to solve Eq. (25), Let $\boldsymbol{X}=\boldsymbol{D} \boldsymbol{\alpha}$, Eq. (25) can be rewritten as

$$
\min _{\boldsymbol{\alpha}} \boldsymbol{L}_{2}(\boldsymbol{\alpha})=\min _{\boldsymbol{\alpha}} \frac{1}{2}\|\boldsymbol{X}-\boldsymbol{R}\|_{2}^{2}+\frac{\lambda}{\rho}\|\boldsymbol{w} \boldsymbol{\alpha}\|_{p}
$$

To enable a tractable solution of Eq. (26), in this paper, a general assumption is made, with which even a closed form can be achieved. Specifically, $\boldsymbol{R}$ can be regarded as some type of noisy observation of $\boldsymbol{X}$, and then the assumption is made that each element of $\boldsymbol{E}=\boldsymbol{X}-\boldsymbol{R}$ follows an independent zeromean distribution with variance $\sigma^{2}$. The following conclusion can be proved by this assumption.

Theorem 3 Define $\boldsymbol{X}, \boldsymbol{R} \in \Re^{N}, \boldsymbol{X}_{i}, \boldsymbol{R}_{i}$, and $\boldsymbol{e}(j)$ as each element of error vector $\boldsymbol{e}$, where $\boldsymbol{e}=\boldsymbol{X}-\boldsymbol{R}, j=1, \ldots, N$. Assume that $\boldsymbol{e}(j)$ follows an independent zero mean distribution with variance $\sigma^{2}$, and thus for any $\varepsilon>0$, we can represent the relationship between $\frac{1}{N}\|\boldsymbol{X}-\boldsymbol{R}\|_{2}^{2}$ and $\frac{1}{K} \sum_{i=1}^{n}\left\|\boldsymbol{X}_{i}-\boldsymbol{R}_{i}\right\|_{2}^{2}$ by the following property,

$$
\lim _{\substack{N \rightarrow \infty \\ K \rightarrow \infty}} \boldsymbol{P}\left\{\left|\frac{1}{N}\|\boldsymbol{X}-\boldsymbol{R}\|_{2}^{2}-\frac{1}{K} \sum_{i=1}^{n}\right|\left|\boldsymbol{X}_{i}-\boldsymbol{R}_{i} \|_{F}^{2}\right|<\varepsilon\right\}=1
$$

where $\boldsymbol{P}(\bullet)$ represents the probability and $K=d \times k \times n$. The detailed proof of Theorem 3 is given in supplemental matarial.

Based on Theorem 3, we have the following equation with a very large probability (restricted 1) at each iteration,

$$
\frac{1}{N}\|\boldsymbol{X}-\boldsymbol{R}\|_{2}^{2}=\frac{1}{K} \sum_{i=1}^{n}\left\|\boldsymbol{X}_{i}-\boldsymbol{R}_{i}\right\|_{F}^{2}
$$

Based on Eqs. (26) and (28), we have

$$
\begin{aligned}
& \min _{\boldsymbol{\alpha}} \frac{1}{2}\|\boldsymbol{X}-\boldsymbol{R}\|_{2}^{2}+\frac{\lambda}{\rho}\left\|\boldsymbol{w}_{\boldsymbol{\alpha}}\right\|_{p} \\
& =\min _{\boldsymbol{\alpha}_{i}}\left(\sum_{i=1}^{n} \frac{1}{2}\left\|\boldsymbol{X}_{i}-\boldsymbol{R}_{i}\right\|_{F}^{2}+\tau_{i}\left\|w_{i} \boldsymbol{\alpha}_{i}\right\|_{p}\right) \\
& =\min _{\boldsymbol{\alpha}_{i}}\left(\sum_{i=1}^{n} \frac{1}{2}\left\|\boldsymbol{R}_{i}-\boldsymbol{D}_{i} \boldsymbol{\alpha}_{i}\right\|_{F}^{2}+\tau_{i}\left\|w_{i} \boldsymbol{\alpha}_{i}\right\|_{p}\right)
\end{aligned}
$$

where $\tau_{i}=\lambda_{i} K / \rho N$ and $\boldsymbol{D}_{i}$ is a dictionary. Clearly, Eq. (29) can be viewed as a sparse coding problem by solving $n$ subproblems for all the group $\boldsymbol{X}_{i}$. Based on Theorem 1, Eq. (29) can be rewritten as:

$$
\hat{\boldsymbol{\alpha}}_{i}=\min _{\boldsymbol{\alpha}_{i}} \sum_{i=1}^{n} \frac{1}{2}\left\|\boldsymbol{\gamma}_{i}-\boldsymbol{\alpha}_{i}\right\|_{2}^{2}+\tau_{i}\left\|w_{i} \boldsymbol{\alpha}_{i}\right\|_{p}
$$

where $\boldsymbol{R}_{i}=\boldsymbol{D}_{i} \gamma_{i}$ and $\boldsymbol{X}_{i}=\boldsymbol{D}_{i} \boldsymbol{\alpha}_{i}$

To obtain the solution of Eq. (30) effectively, in this paper, the generalized soft-thresholding (GST) algorithm [16] is used to solve Eq. (30). Therefore, a closed-form solution of Eq. (30) can be computed as

$$
\hat{\boldsymbol{\alpha}}_{i}=G S T\left(\gamma_{i}, \tau_{i} w_{i}, p\right)
$$

For more details about the GST algorithm, please refer to [16]. For each weight $w_{i}$, large values of sparse coefficient $\boldsymbol{\alpha}_{i}$ usually transmit major edge and texture information. 
Table 1. ADMM method for the Proposed Scheme.

Input: the observed image $\boldsymbol{Y}$ and the measurement matrix $\boldsymbol{H}$.

Initialization: $t, \boldsymbol{C}, \boldsymbol{Z}, \boldsymbol{\alpha}, I, d, k, \rho, p, \sigma, \epsilon, \varepsilon$;

Repeat

Update $\boldsymbol{Z}^{t+1}$ by Eq. (24);

$\boldsymbol{R}^{t+1}=\boldsymbol{Z}^{t+1}-\boldsymbol{C}^{t}$;

For $\quad$ Each group $\boldsymbol{R}_{i}$;

Construct dictionary $\boldsymbol{D}_{i}$ by computing Eq. (9);

Update $\lambda_{i}{ }^{t+1}$ by computing $\lambda_{i}=2 \sqrt{2} \sigma^{2} / \delta_{i}+\varepsilon$;

Update $\tau_{i}^{t+1}$ computing by $\tau_{i}=\lambda_{i} K / \rho N$;

Update $w_{i}^{t+1}$ computing by $w_{i}=\tau_{i} /\left|\gamma_{i}\right|+\epsilon$;

Update $\boldsymbol{\alpha}_{i}^{t+1}$ computing by Eq. (31);

End For

Update $\boldsymbol{D}^{t+1}$ by concatenating all $\boldsymbol{D}_{i}$;

Update $\boldsymbol{\alpha}^{t+1}$ by concatenating all $\boldsymbol{\alpha}_{i}$;

Update $\boldsymbol{C}^{t+1}$ by computing Eq. (22) ;

$t \leftarrow t+1$

Until

maximum iteration number is reached.

Output:

The final restored image $\hat{\boldsymbol{X}}=\boldsymbol{D} \hat{\boldsymbol{\alpha}}$.

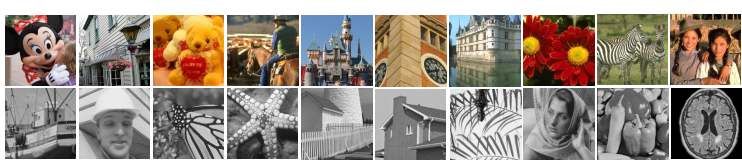

Fig. 2. All test images.

This implies that to reconstruct $\boldsymbol{X}_{i}$ from its degraded one, we should shrink large values less, while shrinking smaller ones more, and thus we have $w_{i}=1 /\left(\left|\gamma_{i}\right|+\epsilon\right)$, where $\epsilon$ is a small constant. Inspired by [17], the regularization parameter $\lambda_{i}$ of each group $\boldsymbol{R}_{i}$ is set as: $\lambda_{i}=2 \sqrt{2} \sigma^{2} /\left(\delta_{i}+\varepsilon\right)$, where $\delta_{i}$ denotes the estimated variance of $\gamma_{i}$, and $\varepsilon$ is a small constant. After solving the two sub-problems, we summarize the overall algorithm for Eq. (19) in Table 1.

\section{EXPERIMENTAL RESULTS}

In this section, we report our experimental results in the applications of image inpainting and image CS recovery. All the experimental images are shown in Fig. 2. The Matlab code can be download at: https://drive.google.com/ open? id=0B0wKhHwcknCjS115YnM1RFhIVUk.

In image inpainting, two interesting examples of image inpainting with different masks are conducted, i.e., image restoration from partial random samples and text inlayed sample. The parameters are set as follows. The size of each patch $\sqrt{d} \times \sqrt{d}$ is set to be $8 \times 8$ and $10 \times 10$ for partial random samples and text inlayed, respectively. Similar patch number$\mathrm{s} k=60, I=25, \sigma=\sqrt{2}, \epsilon=0.1, \varepsilon=0.3 .(\rho, p)$ are set to $(0.0003,0.45),(0.0003,0.45),(0.03,1),(0.04,1)$ and

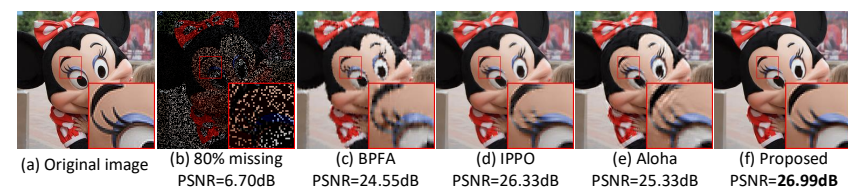

Fig. 3. Visual comparison of Mickey by image inpainting with $80 \%$ missing pixels.

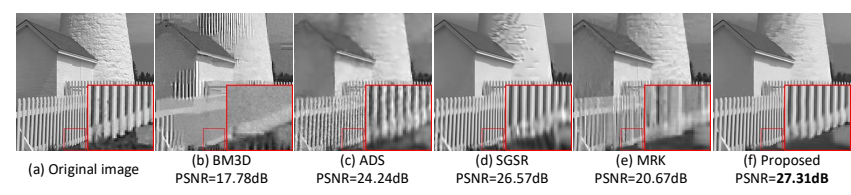

Fig. 4. Visual comparison of Fence by $\mathrm{CS}$ recovery with $0.1 \mathrm{~N}$ measurements.

$(0.06,0.95)$ when $80 \%, 70 \%, 60 \%, 50 \%$ pixels missing and text inlayed, respectively.

We have compared the proposed scheme with three other competing methods: BPFA method [2], IPPO method [18], Aloha method [19]. Table 2 lists the average PSNR comparison results for a collection of 10 color images among three competing methods. The visual comparison of the image inpainting methods is shown in Fig. 3. It can be seen that BPFA could not reconstruct sharp edges and fine details. The IPPO and Aloha methods produce images with a much better visual quality than BPFA method, but still suffer from some undesirable artifacts, such as the ringing effects. The proposed scheme not only preserves sharper edges and finer details, but eliminates the ringing effects.

In image CS recovery, we generate the CS measurements at the block level by utilizing a Gaussian random projection matrix to test images, i.e., the CS recovery with block size $32 \times 32$. The parameters are set as follows. The size of each patch $\sqrt{d} \times \sqrt{d}$ is set to be $7 \times 7$. Similar patch numbers $k=60, I=20, \sigma=\sqrt{2}, \epsilon=0.1, \varepsilon=0.4$. $(\rho, p)$ are set to $(0.0001,0.65),(0.0005,0.5)$ and $(0.05,1)$ when $0.1 N, 0.2 N$ and $0.3 \mathrm{~N}$ measurements, respectively.

We have compared the proposed method based image CS recovery against four other competing approaches including BM3D method [20], ADS method [3], SGSR method [21] and MRK method [22]. The average PSNR results are shown in Table 3. The average gain of the proposed scheme over BM3D, ADS, SGSR and MRK methods can be as much as $3.31 \mathrm{~dB}, 1.04 \mathrm{~dB}, 1.24 \mathrm{~dB}$ and $2.11 \mathrm{~dB}$, respectively. The visual comparison of the image CS recovery is shown in Fig. 4. It can be seen that the BM3D, ADS, SGSR, and MRK methods still suffer from some undesirable artifacts or over-smooth phenomena. By contrast, the proposed method not only removes most of the visual artifacts, but also preserves largescale sharp edges and small-scale fine image details more effectively. 
Table 2. Average PSNR results (dB) of image inpainting

\begin{tabular}{|c|c|c|c|c|c|}
\hline Method & $\mathbf{8 0 \%}$ & $\mathbf{7 0 \%}$ & $\mathbf{6 0 \%}$ & $\mathbf{5 0 \%}$ & Inlay text \\
\hline BPFA & 24.35 & 26.26 & 28.03 & 29.83 & 31.42 \\
\hline IPPO & 25.40 & 27.34 & 29.19 & 30.93 & 32.90 \\
\hline Aloha & 25.13 & 26.92 & 28.51 & 30.13 & 31.12 \\
\hline Proposed & $\mathbf{2 5 . 9 1}$ & $\mathbf{2 8 . 0 4}$ & $\mathbf{2 9 . 9 8}$ & $\mathbf{3 1 . 9 5}$ & $\mathbf{3 3 . 5 8}$ \\
\hline
\end{tabular}

Table 3. Average PSNR results (dB) of image CS recovery

\begin{tabular}{|c|c|c|c|c|c|}
\hline Ratio & BM3D & ADS & SGSR & MRK & Proposed \\
\hline 0.1 & 24.23 & 27.65 & 27.93 & 27.14 & $\mathbf{2 8 . 8 9}$ \\
\hline 0.2 & 30.60 & 32.42 & 32.36 & 31.22 & $\mathbf{3 3 . 5 0}$ \\
\hline 0.3 & 34.11 & 35.68 & 34.87 & 34.20 & $\mathbf{3 6 . 5 0}$ \\
\hline
\end{tabular}

\section{CONCLUSION}

This paper analyzed the group sparsity based on the rank minimization methods. An adaptive dictionary learning method for each group was designed. We proved the equivalence of the group-based sparse coding and the rank minimization problem, and thus the sparse coefficients of each group were measured by computing the singular values of each group. Four nuclear norms were used to analyze the sparsity of each group and the solution of the weighted Schatten $p$-norm minimization (WSNM) is the best approximation to real singular values of each group. Therefore, WSNM can be equivalently transformed into a non-convex $\ell_{p}$-norm minimization problem in group-based sparse coding. Experimental results on image inpainting and image CS recovery have demonstrated that the proposed scheme achieves significant performance improvements over the current state-of-the-art methods.

\section{ACKNOWLEDGE}

The authors would like to thank Dr. Jian Zhang ${ }^{1}$ of Peking University for his help. In addition, this work was supported by the NSFC $(61571220,61462052,61502226)$ and the open research fund of National Mobile Commune. Research Lab., Southeast University (No.2015D08).

\section{REFERENCES}

[1] Michael Elad and Michal Aharon, "Image denoising via sparse and redundant representations over learned dictionaries," IEEE Transactions on Image processing, vol. 15, no. 12, pp. 3736-3745, 2006.

[2] Mingyuan Zhou, Haojun Chen, Lu Ren, Guillermo Sapiro, Lawrence Carin, and John W Paisley, "Non-parametric bayesian dictionary learning for sparse image representations," in Advances in neural information processing systems, 2009, pp. 2295-2303.

[3] Weisheng Dong, Guangming Shi, Xin Li, Lei Zhang, and Xiaolin Wu, "Image reconstruction with locally adaptive sparsity and nonlocal robust regularization," Signal Processing: Image Communication, vol. 27, no. 10, pp. 1109-1122, 2012.

\footnotetext{
${ }^{1}$ http://124.207.250.90/staff/zhangjian/
}

[4] Jian Zhang, Debin Zhao, and Wen Gao, "Group-based sparse representation for image restoration," IEEE Transactions on Image Processing, vol. 23, no. 8, pp. 3336-3351, 2014.

[5] Julien Mairal, Francis Bach, Jean Ponce, Guillermo Sapiro, and Andrew Zisserman, "Non-local sparse models for image restoration," in Proc. IEEE Int. Conf. Comput. Vis. IEEE, 2009, pp. 2272-2279.

[6] Emmanuel J Candes, Michael B Wakin, and Stephen P Boyd, "Enhancing sparsity by reweighted $\ell_{1}$ minimization," Journal of Fourier analysis and applications, vol. 14, no. 5-6, pp. 877-905, 2008.

[7] Emmanuel J Candès, Justin Romberg, and Terence Tao, "Robust uncertainty principles: Exact signal reconstruction from highly incomplete frequency information," IEEE Transactions on information theory, vol. 52, no. 2, pp. 489-509, 2006.

[8] Jian-Feng Cai, Emmanuel J Candès, and Zuowei Shen, "A singular value thresholding algorithm for matrix completion," SIAM Journal on Optimization, vol. 20, no. 4, pp. 1956-1982, 2010.

[9] Shuhang Gu, Qi Xie, Deyu Meng, Wangmeng Zuo, Xiangchu Feng, and Lei Zhang, "Weighted nuclear norm minimization and its applications to low level vision," International Journal of Computer Vision, pp. 1-26, 2016.

[10] Feiping Nie, Heng Huang, and Chris HQ Ding, "Low-rank matrix recovery via efficient schatten p-norm minimization.," in AAAI, 2012.

[11] Y. Xie, S. Gu, Y. Liu, W. Zuo, W. Zhang, and L. Zhang, "Weighted schatten $\mathrm{p}$-norm minimization for image denoising and background subtraction," IEEE Transactions on Image Processing, vol. 25, no. 10, pp. 4842-4857, Oct 2016.

[12] Qifa Ke and Takeo Kanade, "Robust $\ell_{1}$ norm factorization in the presence of outliers and missing data by alternative convex programming," in 2005 IEEE Computer Society Conference on Computer Vision and Pattern Recognition (CVPR'05). IEEE, 2005, vol. 1, pp. 739-746.

[13] Anders P Eriksson and Anton Van Den Hengel, "Efficient computation of robust low-rank matrix approximations in the presence of missing data using the 11 norm.," in $C V P R, 2010$, vol. 1, p. 4.

[14] Chengbo Li, Wotao Yin, and Yin Zhang, "Users guide for tval3: Tv minimization by augmented lagrangian and alternating direction algorithms," CAAM report, vol. 20, pp. 46-47, 2009.

[15] Stephen Boyd, Neal Parikh, Eric Chu, Borja Peleato, and Jonathan Eckstein, "Distributed optimization and statistical learning via the alter-

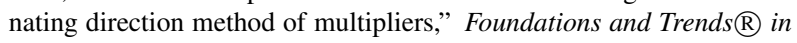
Machine Learning, vol. 3, no. 1, pp. 1-122, 2011.

[16] Wangmeng Zuo, Deyu Meng, Lei Zhang, Xiangchu Feng, and David Zhang, "A generalized iterated shrinkage algorithm for non-convex sparse coding," in Proceedings of the IEEE international conference on computer vision, 2013, pp. 217-224.

[17] S Grace Chang, Bin Yu, and Martin Vetterli, "Adaptive wavelet thresholding for image denoising and compression," IEEE Trans. Image Process., vol. 9, no. 9, pp. 1532-1546, 2000.

[18] Idan Ram, Michael Elad, and Israel Cohen, "Image processing using smooth ordering of its patches," IEEE transactions on image processing, vol. 22, no. 7, pp. 2764-2774, 2013.

[19] Kyong Hwan Jin and Jong Chul Ye, "Annihilating filter-based low-rank hankel matrix approach for image inpainting," IEEE Transactions on Image Processing, vol. 24, no. 11, pp. 3498-3511, 2015.

[20] Karen Egiazarian, Alessandro Foi, and Vladimir Katkovnik, "Compressed sensing image reconstruction via recursive spatially adaptive filtering," in 2007 IEEE International Conference on Image Processing. IEEE, 2007, vol. 1, pp. I-549.

[21] Jian Zhang, Debin Zhao, Feng Jiang, and Wen Gao, "Structural group sparse representation for image compressive sensing recovery," in Data Compression Conference (DCC), 2013. IEEE, 2013, pp. 331-340.

[22] Thuong Nguyen Canh, Khanh Quoc Dinh, and Byeungwoo Jeon, "Multi-scale/multi-resolution kronecker compressive imaging," in Image Processing (ICIP), 2015 IEEE International Conference on. IEEE, 2015, pp. 2700-2704. 